J. Lake Sci.(湖泊科学), 2017, 29(2): 308-316

DOI 10. 18307/2017. 0206

(c) 2017 by Journal of Lake Sciences

\title{
云南阳宗海表层沉积物有机质组成结构对磷赋存形态特征的影响”
}

\author{
赵忠红 ${ }^{1}$, 张乃明 ${ }^{1 * *}$, 扈学文 ${ }^{2,3}$,包 力 $^{1}$, 夏运生 ${ }^{1}$ \\ ( 1 : 云南农业大学资源与环境学院, 昆明 650201) \\ (2: 中国环境科学研究院,环境基准与风险评估国家重点实验室,北京 100012) \\ (3: 中国环境科学研究院国家环境保护湖泊污染控制重点实验室,湖泊生态环境创新基地, 北京 100012)
}

\begin{abstract}
摘 要: 利用连续提取分级的方法定量分析阳宗海表层沉积物磷赋存形态, 阐明了沉积物 C、N、H 和 O 组成及溶解有机 质 (DOM) 紫外-可见光谱特征, 探讨沉积物元素组成及 DOM 组成结构对不同形态磷含量的影响. 结果表明: (1) 沉积物潜 在可移动磷含量在 68.67 124.70 mg/kg 之间变化, 平均占总磷含量的 9.81\%, 表现为 BD-P $>\mathrm{NaOH}-\mathrm{nrP}>\mathrm{NH}_{4} \mathrm{Cl}-\mathrm{P}$; 沉积 物稳定磷含量在 496.73 908.28 mg/ $\mathrm{kg}$ 之间变化, 平均占总磷含量的 60.86\%. (2) 沉积物 C、 $\mathrm{N}$ 含量和疏水性 DOM 光谱参 数 $\mathrm{A}_{240-400}$ 表现出北部高、南部低的变化趋势, 但 $\mathrm{H} / \mathrm{C} 、 \mathrm{O} / \mathrm{C}$ 和 $(\mathrm{N}+\mathrm{O}) / \mathrm{C}$ 摩尔比和亲水性 $D O M$ 光谱参数 $\mathrm{A}_{240-400}$ 变化趋势 则与之恰好相反. (3) 沉积物 $\mathrm{NH}_{4} \mathrm{Cl}-\mathrm{P}$ 含量与 $\mathrm{C} 、 \mathrm{~N}$ 和 $\mathrm{H}$ 含量之间呈显著正相关, 但与 $\mathrm{H} / \mathrm{C} 、 \mathrm{O} / \mathrm{C} 、(\mathrm{~N}+\mathrm{O}) / \mathrm{C}$ 摩尔比和亲水 性 DOM 光谱参数 $\mathrm{E}_{2} / \mathrm{E}_{3}$ 值之间呈显著负相关; $\mathrm{NaOH}-\mathrm{rP}$ 和 BD-P $+\mathrm{NaOH}-\mathrm{rP}$ 含量均与 $\mathrm{O}$ 含量及 $\mathrm{O} / \mathrm{H}$ 摩尔比呈显著负相 关; NaOH-rP、BD-P+NaOH-rP 和 HCl-P 均与疏水性 DOM 光谱参数 $\mathrm{A}_{240-400}$ 值之间呈显著正相关. 因此, 天然有机质元素组 成及官能团结构是影响沉积物磷赋存形态的重要因素.
\end{abstract}

关键词: 阳宗海;沉积物;元素组成; 溶解有机质; 磷形态

\section{Effects of composition and structure of natural organic matter on phosphorus fractions in sediment from Lake Yangzonghai, Yunnan Province}

ZHAO Zhonghong ${ }^{1}$, ZHANG Naiming ${ }^{1 * *}$, HU Xuewen ${ }^{2,3}$, BAO Li ${ }^{1} \&$ XIA Yunsheng ${ }^{1}$

(1: College of Resources and Environment, Yunnan Agricultural University, Kunming 650201,P.R.China)

(2: State Key Laboratory of Environmental Criteria and Risk Assessment, Chinese Research Academy of Environmental Sciences, Beijing 100012, P.R.China)

(3: Research Center of Lake Eco-Environment, State Environmental Protection Key Laboratory for Lake Pollution Control, Chinese Research Academy of Environmental Sciences, Beijing 100012, P.R. China)

Abstract: Characteristics of phosphorus fractions in surface sediments of the Lake Yangzonghai were studied applying the method of continuous extraction and classification. The element composition of $\mathrm{C}, \mathrm{N}, \mathrm{H}$ and $\mathrm{O}, \mathrm{UV}$-vis spectrum features of hydrophobic and hydrophilic dissolved organic matter (DOM), and their effects on P fractions in sediments were also clarified.The results indicated that the mobile-P contents in sediment ranged in $68.67-124.70 \mathrm{mg} / \mathrm{kg}$ and accounted for $9.81 \%$ of total phosphorus on average. The immobile-P contents in sediment ranged in $496.73-908.28 \mathrm{mg} / \mathrm{kg}$, accounting for $60.86 \%$ of total phosphorus on average. The contents of different $\mathrm{P}$ forms decreased from north to south part, and the immobile-P content was significantly higher than that of mobile-P, indicating $\mathrm{P}$ retention in sediments. The contents of $\mathrm{C}, \mathrm{N}$ and $\mathrm{UV}$-vis spectrum integral area $\left(\mathrm{A}_{240-400}\right)$ of hydrophilic DOM showed a decreasing trend from north to south of the lake, while the ratios of $\mathrm{H} / \mathrm{C}, \mathrm{O} / \mathrm{C},(\mathrm{O}+\mathrm{N}) / \mathrm{C}$ and the $\mathrm{A}_{240-400}$ value of hydrophobic DOM presented a reversing trend. Significantly positive correlations were found between $\mathrm{NH}_{4} \mathrm{Cl}-\mathrm{P}$ and $\mathrm{C}, \mathrm{N}$ and $\mathrm{H}$ contents, and the negative correlations were found between $\mathrm{NH}_{4} \mathrm{Cl}-\mathrm{P}$ contents and $\mathrm{H} / \mathrm{C}, \mathrm{O} / \mathrm{C},(\mathrm{N}+\mathrm{O}) / \mathrm{C}$, UV-vis spectrum eigenratio $\left(\mathrm{E}_{2} / \mathrm{E}_{3}\right)$ of hydrophilic DOM. In addition, $\mathrm{NaOH}-\mathrm{rP}$ and $\mathrm{BD}-\mathrm{P}+\mathrm{NaOH}-\mathrm{rP}$ have negative correlations with the $\mathrm{O}$ and $\mathrm{O} / \mathrm{H}$ ratios, but all of the NaOH-rP, BD-P+NaOH-rP and HCl-P contents in sediments have positive correlations with the $\mathrm{A}_{240-400}$ value

* 云南省科技创新人才计划 (2015HC018) 项目资助. 2016-03-07 收稿; 2016-05-31 收修改稿. 赵忠红(1979 ), 女,硕士研究生;E-mail: 79457748@ qq.com.

** 通信作者;E-mail:zhangnaiming@ sina.com. 
of hydrophobic DOM. Therefore, element composition and functional groups structure of nature organic matter is the important factors influencing the $\mathrm{P}$ fractions.

Keywords: Lake Yangzonghai; sediment; elements composition; dissolved organic matter; phosphorus fractions

沉积物磷释放是湖泊水体中磷的重要来源之一,尤其在外源磷人湖负荷得到控制后, 沉积物内源磷释 放在一定程度上较长时间内将影响着水体的富营养化水平 ${ }^{[1-4]}$. 弱吸附态磷 $\left(\mathrm{NH}_{4} \mathrm{Cl}-\mathrm{P}\right)$ 、氧化还原敏感磷 (BD-P) 和生物有机磷 ( NaOH-nrP) 被认为是沉积物潜在可移动磷形态 (Mobile-P), 而铝结合态磷 ( NaOH$r P)$ 、钲结合态磷 (HCl-P) 和残渣磷 (Res-P) 则被认为是沉积物稳定磷形态 (Immobile-P $)^{[5-6]}$. Reitzel 等 ${ }^{[6]}$ 研 究认为, Mobile-P 在较短时间尺度即可周转循环, 其半衰期大约为 $10 \mathrm{a}$, 而 Immobile-P 则在较长时间尺度才 能周转循环, 其半衰期大约为 $100 \mathrm{a}$. 很多研究表明, 有机质和金属化合物均是影响沉积物磷赋存形态的重要 因素, $\mathrm{NH}_{4} \mathrm{Cl}-\mathrm{P}$ 含量与沉积物有机质和 $\mathrm{CaCO}_{3}$ 密切相关 ${ }^{[4,7-9]}$, 随着铁、铝和钙等金属化合物和有机质含量的 提高, 沉积物中 $\mathrm{Fe} / \mathrm{Al}-\mathrm{P}$ 和 $\mathrm{Ca}-\mathrm{P}$ 含量也显著增加 ${ }^{[10-13]}$. 关于有机质和金属化合物对磷吸附/释放特征的影响 国际上已有较多报道 ${ }^{[14-18]}$. 有机质矿化降解过程中可使沉积物/水界面氧化还原电位降低,作为电子受体的 $\mathrm{MnO}_{2}$ 和 $\mathrm{FeOOH}$ 等被还原成溶解性的 $\mathrm{Mn}^{2+}$ 和 $\mathrm{Fe}^{2+}$, 从而导致铁锰结合的磷形态大量释放出来 ${ }^{[14-15]}$. 研究发 现, 低分子量有机酸能够活化结晶铁铝氧化物和阻碍无定形铁铝氧化物结晶, 从而促进了磷的吸附作用 ${ }^{[16]}$, 土壤中磷吸附能力的提高与有机质分解产生低分子量有机化合物通过与金属键合, 形成具有稳定化学结构 的有机一金属鳌合体有关 ${ }^{[17]}$. 但也有研究报道, 由于腐殖酸类物质 $(\mathrm{HA})$ 占据氧化铁的表面吸附位点, 从而降 低了磷的吸附能力 ${ }^{[18-19]}$. Wang 等 ${ }^{[20-21]}$ 研究表明, 沉积物粒度、有机质和离子强度均显著影响磷的吸附作用, 沉积物轻组有机质 (LFOM) 去除后, 磷酸盐吸附量的下降主要与羧基、羟基等官能团结构减少有关. 可见,有 机质在沉积物磷赋存形态及吸附/释放过程中扮演着非常重要的作用. 然而, 由于天然有机质具有高度异质 性, 有机质含量及组成结构不同, 其对沉积物磷赋存特征的影响也明显不同. 阐明有机质组成结构对沉积物 磷赋存特征影响, 对揭示沉积物磷滞留及潜在的风险具有重要的指导意义. 另外, 溶解性有机质的官能团能 够与砷酸盐和亚砷酸盐形成络合物, 可降低砷的迁移及生物有效性, 而水体中高浓度磷酸盐则会与砷产生 竞争吸附, 进而促进沉积物砷的释放 ${ }^{[22-23]}$. 因此, 有机质组成结构与沉积物磷和砷赋存之间均具有密切关系. 近年来, 阳宗海砷污染事件广泛受到世界各界人士关注 ${ }^{[24]}$, 通过本项研究, 对进一步揭示阳宗海沉积物砷的 环境风险具有重要的借鉴作用.

\section{1 材料与方法}

\section{1 样品采集与流域特征}

阳宗海 ( $24^{\circ} 27^{\prime} \sim 24^{\circ} 54^{\prime} \mathrm{N}, 102^{\circ} 55^{\prime} \sim 103^{\circ} 2^{\prime} \mathrm{E}$ ) 位于宜良、呈贡、澄江三县交界处, 属于珠江流域南盘江水 系, 流域面积 $286 \mathrm{~km}^{2}$, 湖面面积 $31.9 \mathrm{~km}^{2}$, 最大水深 $29.1 \mathrm{~m}$, 平均水深 $20.0 \mathrm{~m}$. 南部靠近阳宗镇, 人湖河流包 括阳宗大河、七星河和鲁溪冲河, 北部靠近汤池镇, 人湖河流为摆依河和汤池河. 阳宗海工矿企业主要分布 在南岸和北岸, 其中南岸有磷肥厂和耐火厂, 北岸有磷肥厂、火电厂和粉煤灰堆场. 阳宗海是云南高原水质 相对较好的湖泊之一, 水质基本为 II 类或 III 类, 但近年来呈现下降趋势. 农村农业面源是阳宗海流域的主要 污染源, 有 $46 \%$ 的氮磷污染来自于农村农业面源.

2014 年 9 月采集阳宗海由北至南不同点位上覆水和表层沉积物样品, 同时采用水深测定仪和塞氏盘现 场测定水深和透明度, 采集的水样和沉积物样品立即带回实验室, 水体中总氮 ( TN) 和总磷 ( TP) 等水质指标 采用过硫酸钾一分光光度法测定, 沉积物样品冷冻干燥后, 过 100 目篮后备用. 采样点位置及水环境指标见 图 1 和表 1.

\section{2 分析及计算方法}

沉积物 TP 含量采用欧洲标准测试委员会框架下发展的 SMT 法 ${ }^{[25]}$, 沉积物磷形态提取方法采用 Psenner ${ }^{[26]}$ 提出的连续提取法. 沉积物有机元素分析的前处理方法: 将样品中加人 $0.5 \mathrm{~mol} / \mathrm{L}$ 盐酸, 摚拌均匀后放 置 $24 \mathrm{~h}$, 再加人去离子水清洗直至 $\mathrm{pH}$ 值达 6 7, 样品在 $40^{\circ} \mathrm{C}$ 以下烘干后研磨至 200 目备用. 沉积物样品 $\mathrm{C} 、$ $\mathrm{N} 、 \mathrm{H}$ 和 $\mathrm{O}$ 含量采用德国 Elementar 公司 Vario Micro cube 型号元素分析仪分析测定, 重复 3 次. 沉积物溶解 


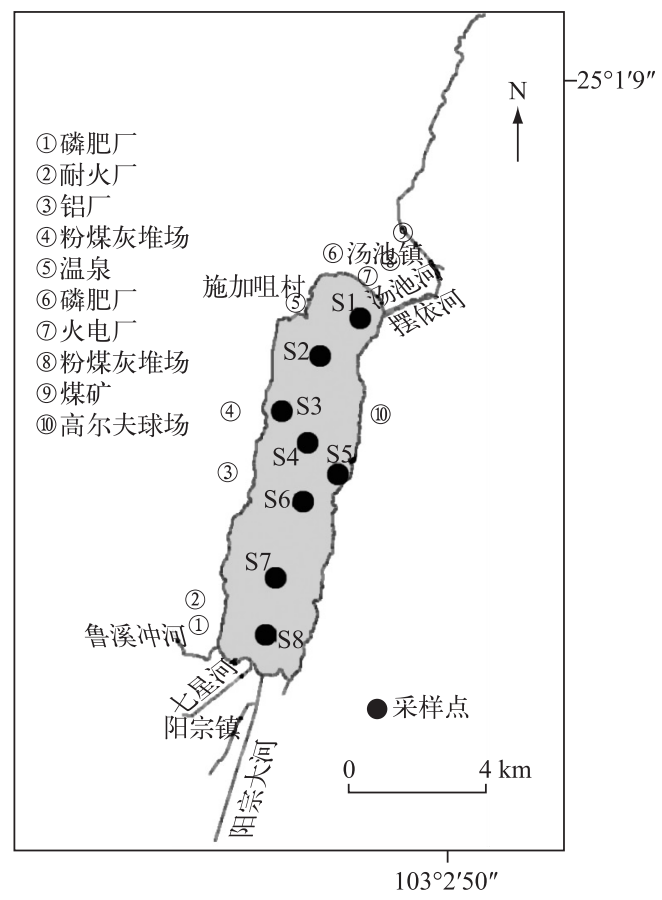

图 1 阳宗海采样点分布

Fig.1 Distribution of sampling sites in Lake Yangzonghai

表 1 阳宗海采样点坐标及水质指标

Tab.1 Geographic coordinates of sampling sites and water quality index in Lake Yangzonghai

\begin{tabular}{ccccccc}
\hline 点位 & 经度 & 纬度 & 水深 $/ \mathrm{m}$ & 透明度 $/ \mathrm{m}$ & $\mathrm{TN} /(\mathrm{mg} / \mathrm{L})$ & $\mathrm{TP} /(\mathrm{mg} / \mathrm{L})$ \\
\hline $\mathrm{S} 1$ & $103^{\circ} 1^{\prime} 10.71^{\prime \prime} \mathrm{E}$ & $24^{\circ} 57^{\prime} 15.98^{\prime \prime} \mathrm{N}$ & 18.60 & 1.26 & 1.27 & 0.06 \\
$\mathrm{~S} 2$ & $103^{\circ} 0^{\prime} 29.20^{\prime \prime} \mathrm{E}$ & $24^{\circ} 56^{\prime} 39.40^{\prime \prime} \mathrm{N}$ & 20.50 & 1.26 & 1.35 & 0.08 \\
$\mathrm{~S} 3$ & $102^{\circ} 59^{\prime} 50.13^{\prime \prime} \mathrm{E}$ & $24^{\circ} 55^{\prime} 45.84^{\prime \prime} \mathrm{N}$ & 19.70 & 1.25 & 1.34 & 0.07 \\
$\mathrm{~S} 4$ & $103^{\circ} 0^{\prime} 17.27^{\prime \prime} \mathrm{E}$ & $24^{\circ} 55^{\prime} 15.54^{\prime \prime} \mathrm{N}$ & 22.10 & 1.32 & 1.16 & 0.07 \\
$\mathrm{~S} 5$ & $103^{\circ} 0^{\prime} 49.27^{\prime \prime} \mathrm{E}$ & $24^{\circ} 54^{\prime} 46.09^{\prime \prime} \mathrm{N}$ & 23.40 & 1.25 & 1.31 & 0.05 \\
$\mathrm{~S} 6$ & $103^{\circ} 0^{\prime} 13.95^{\prime \prime} \mathrm{E}$ & $24^{\circ} 54^{\prime} 18.94^{\prime \prime} \mathrm{N}$ & 23.10 & 1.30 & 1.08 & 0.07 \\
$\mathrm{~S} 7$ & $102^{\circ} 59^{\prime} 46.42^{\prime \prime} \mathrm{E}$ & $24^{\circ} 53^{\prime} 4.96^{\prime \prime} \mathrm{N}$ & 21.50 & 1.33 & 1.11 & 0.08 \\
$\mathrm{~S} 8$ & $102^{\circ} 59^{\prime} 36.98^{\prime \prime} \mathrm{E}$ & $24^{\circ} 52^{\prime} 10.51^{\prime \prime} \mathrm{N}$ & 19.50 & 1.35 & 1.05 & 0.06 \\
\hline
\end{tabular}

有机质 (DOM) 分组采用 XAD-8 树脂分离法 ${ }^{[27]}$, 具体为: XAD-8 树脂在 $0.1 \mathrm{~mol} / \mathrm{L} \mathrm{NaOH}$ 中浸泡 $24 \mathrm{~h}$, 用丙酮 和正己烷各抽提 $12 \mathrm{~h}$ 以除去有机物, 然后浸泡于甲醇中装柱. 树脂柱高 $50 \mathrm{~cm}$, 直径 $4.5 \mathrm{~cm}$, 树脂粒径 $50 \sim$ $250 \mu \mathrm{m}$, 用甲醇洗去柱中的丙酮和正己烷, 最后用超纯水洗净甲醇直至出水溶解性有机碳 ( DOC) 浓度小于 $1 \mathrm{~mol} / \mathrm{L}$. 将提取的 DOM 以 $1 \mathrm{ml} / \mathrm{min}$ 的流速过 XAD-8 树脂柱, 用 $1 \sim 2$ 倍柱体积的超纯水洗净, 收集通过树 脂柱的部分再用 $6 \mathrm{~mol} / \mathrm{L}$ 的 $\mathrm{HCl}$ 调 $\mathrm{pH}$ 至 2 , 用 1 倍柱体积 $0.01 \mathrm{~mol} / \mathrm{L}$ 的 $\mathrm{HCl}$ 淋洗, 即得到亲水组分; 再用 0.25 倍柱体积, $0.1 \mathrm{~mol} / \mathrm{L}$ 的 $\mathrm{NaOH}$ 溶液反洗树脂柱, 接着用 1.5 倍柱体积超纯水洗, 收集反洗液, 即得到疏水 组分.

\section{3 数据统计分析}

数据统计分析及制图采用 Origin 8.5 和 ArcGIS 10.0 软件. 


\section{2 结果与讨论}

\section{1 表层沉积物磷赋存形态特征}

阳宗海表层沉积物 Mobile-P 含量由北至南呈现下降趋势 (图 2), Mobile-P 含量在 68.67 124.70 mg $/ \mathrm{kg}$ 之间变化, 平均为 $91.64 \mathrm{mg} / \mathrm{kg}$. BD-P 是沉积物潜在可移动磷的最重要组成部分, 主要为铁结合态磷, BD-P 释放主要受沉积物一水界面氧化还原条件影响, 当水体厌氧时, 沉积物 BD-P 大量释放, 进而显著影响上覆水 磷浓度 ${ }^{[10,12-13]}$. NaOH-nrP 主要来源于生物有机体的沉积,包括腐殖酸结合磷、磷酸单脂、磷酸二脂、焦磷酸 和多聚磷酸等有机磷化合物. 通常情况下, $\mathrm{NaOH}-\mathrm{nr} P$ 能够矿化为正磷酸盐成为沉积物内源磷负荷的潜在来 源 ${ }^{[28]} \cdot \mathrm{NH}_{4} \mathrm{Cl}-\mathrm{P}$ 是沉积物最活跃的磷形态组分, 尽管沉积物中 $\mathrm{NH}_{4} \mathrm{Cl}-\mathrm{P}$ 含量较低, 不足 $\mathrm{TP}$ 含量的 $1 \%$, 但由 于它是沉积物-水界面磷循环的直接贡献者, 并且在环境条件变化时极容易重新进人水体, 因此, 这部分磷 通常被认为沉积物一水界面磷释放通量的重要指示指标. 本研究潜在可移动磷中 BD-P 含量最高, 在 50.59 $81.24 \mathrm{mg} / \mathrm{kg}$ 之间变化, 平均占 Mobile-P 含量的 66.73\%; NaOH-nrP 含量次之, 在 $16.50 \sim 54.51 \mathrm{mg} / \mathrm{kg}$ 之间变 化, 平均占 Mobile-P 含量的 $31.96 \% ; \mathrm{NH}_{4} \mathrm{Cl}-\mathrm{P}$ 含量最低, 在 $0.32 \sim 1.83 \mathrm{mg} / \mathrm{kg}$ 之间变化, 平均占 Mobile-P 含 量的 $1.31 \%$. 总体而言, BD-P 对 Mobile-P 的贡献呈现南部高、北部低的变化趋势, 而 NaOH-nrP 则与之趋势 相反, 表明北部站点沉积物磷释放主要与有机磷的矿化降解有关, 而南部站点沉积物磷释放则受氧化还原 条件影响较大.

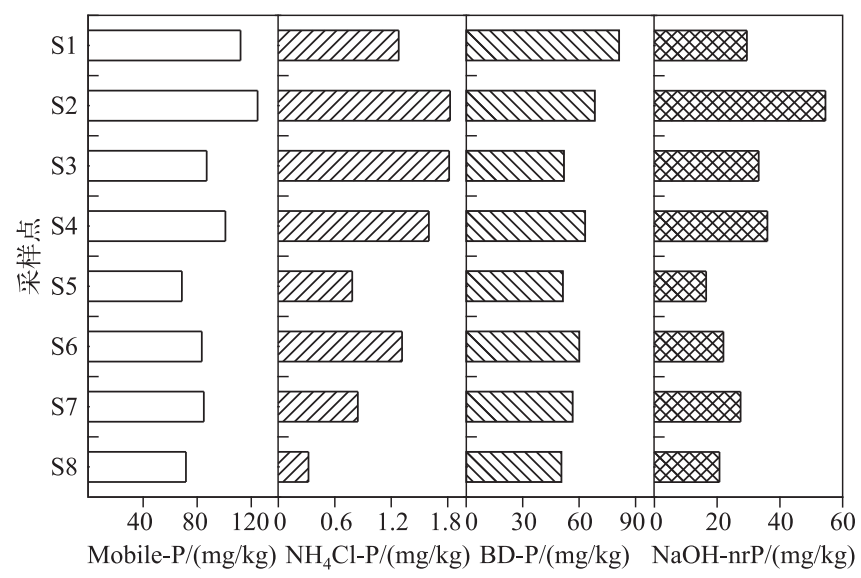

图 2 表层沉积物潜在可移动磷形态空间变化

Fig.2 Spatial changes of Mobile-P fractions in surface sediment

阳宗海表层沉积物 Immobile-P 含量由北向南呈现下降趋势 (图 3), Immobile-P 含量在 496.73 $908.28 \mathrm{mg} / \mathrm{kg}$ 之间变化,平均为 $639.71 \mathrm{mg} / \mathrm{kg}$. NaOH-rP 被公认为较为稳定磷形态的组成部分, 沉积物水合 铝氧化物胶体主要以结晶态和非结晶态两种形式存在, 其中 $\mathrm{Al}(\mathrm{OH})_{3}$ 是主要存在形式, 其不仅具有较高的 吸附能力, 而且可以在好氧和厌氧条件下稳定存在, $\mathrm{NaOH}-\mathrm{rP}$ 主要与 $\mathrm{OH}^{-}$或溶解的磷酸盐复合物发生交换 而释放. 由于 $\mathrm{Al}(\mathrm{OH})_{3}$ 具有较强的吸附能力, 厌氧条件下通常会阻碍 Fe-P 释放, 因此随着 NaOH-rP 增加沉 积物磷滞留越加明显 ${ }^{[10]}$. 作为稳定态磷的重要组成部分 HCl-P 主要来源于陆源输人的碎屑岩以及自生磷灰 石, 通常条件下不易释放, 也难以被生物利用, 只有沉积物呈现弱酸性条件, HCl-P 才逐渐被释放, 而 Res-P 则是最稳定的磷形态, 也被称为惰性磷, 其中大部分为难溶解磷和稳定的有机磷 ${ }^{[4,7]}$. 本研究中 NaOH-rP 含 量在 $159.45 \sim 246.23 \mathrm{mg} / \mathrm{kg}$ 之间变化, 平均占 Immobile-P 的 $31.80 \%$; HCl-P 含量在 $123.24 \sim 391.46 \mathrm{mg} / \mathrm{kg}$ 之 间变化, 平均占 Immobile-P 含量的 $29.51 \%$; Res-P 含量在 $191.32 \sim 297.97 \mathrm{mg} / \mathrm{kg}$ 之间变化, 平均占 Immobile-P 含量的 $38.68 \%$. 其中 NaOH-rP 和 HCl-P 对 Immobile-P 贡献主要表现为南北高、中部低的特点, 而 Res-P 贡献 则与其相反. 表明北部和南部站点以 $\mathrm{NaOH}-\mathrm{rP}$ 和 HCl-P 滞留为主, 受 $\mathrm{pH}$ 值影响较大, 而中部站点则以 Res-P 滞留为主. 


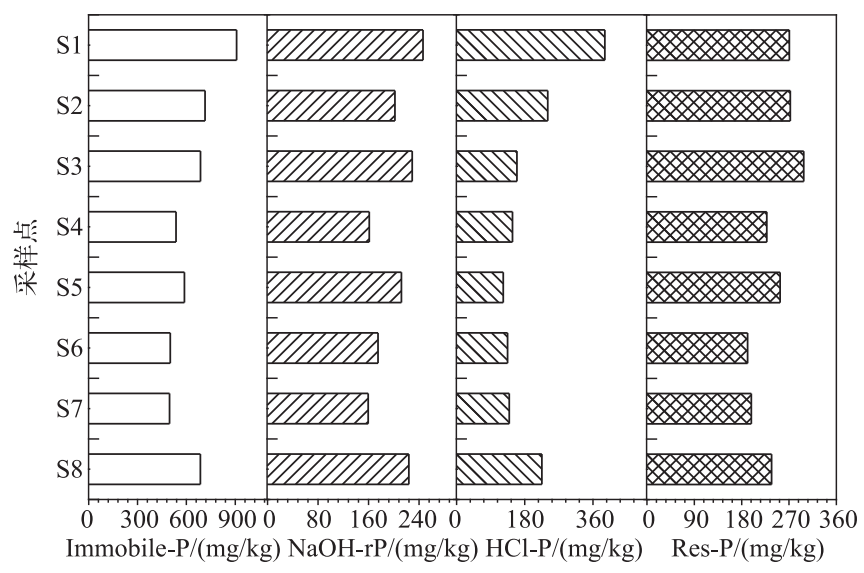

图 3 表层沉积物稳定磷形态空间变化

Fig. 3 Spatial changes of Immobile-P fractions in surface sediment

\section{2 表层沉积物元素组成及 DOM 紫外-可见光谱特征}

沉积物中 $\mathrm{C} 、 \mathrm{~N} 、 \mathrm{H}$ 和 $\mathrm{O}$ 等元素是构成天然有机质的主要指标, 通常可通过 $\delta^{13} \mathrm{C}$ 和 $\delta^{15} \mathrm{~N}$ 稳定同位素、 $\mathrm{C} / \mathrm{N}$ 比及 $\mathrm{C}$ 和 $\mathrm{N}$ 含量变化判断沉积物有机质的来源和丰富 ${ }^{[30]}$. 由于天然有机质具有高度异质性, 不同来 源的有机质组成结构差异较大.随着 $\mathrm{H} / \mathrm{C}$ 比的增大, 有机质中脂肪族化合物增加, 反之, 芳香族化合物则明 显增加 ${ }^{[31]} . \mathrm{O} / \mathrm{C}$ 和 $(\mathrm{O}+\mathrm{N}) / \mathrm{C}$ 比可有效反映有机质的官能团结构, $\mathrm{O} / \mathrm{C}$ 和 $(\mathrm{O}+\mathrm{N}) / \mathrm{C}$ 比越大, 有机质芳香环上 则包含有更多的羰基、羧基、羟基或氨基和硝基等官能团结构, $\mathrm{O} / \mathrm{H}$ 比可有效表征有机质的氧化度, $\mathrm{O} / \mathrm{H}$ 比 越大表明有机质的含氧官能团越多,其氧化度越高, 极性越强, 疏水性则越弱 ${ }^{[31-32]}$.

不同样点沉积物 $\mathrm{C}$ 和 $\mathrm{N}$ 含量分别变化在 1.87\% 5.66\% 和 $0.16 \% \sim 0.33 \%$ 之间, 表现出北部高、南部低的 变化趋势; $\mathrm{H}$ 和 $\mathrm{O}$ 含量分别在 $0.88 \% \sim 1.15 \%$ 和 $8.33 \% \sim 15.86 \%$ 之间变化, 表现为中部高、南北低的变化趋 势; 不同样点沉积物 $\mathrm{H} / \mathrm{C} 、 \mathrm{O} / \mathrm{C} 、(\mathrm{~N}+\mathrm{O}) / \mathrm{C}$ 和 $\mathrm{O} / \mathrm{H}$ 摩尔比分别在 2.27 5.65、1.47 3.42、1.52 3.48 和 $0.56 \sim$ 0.89 之间变化, 与 $\mathrm{C} 、 \mathrm{~N}$ 含量变化趋势恰好相反.表明沉积物有机质含量表现为北部较高、南部较低,脂肪族 化合物及芳香环上具有羰基、羟基和羧基或氨基和硝基等官能团结构的化合物则表现出南部高、北部低的 变化趋势 (表 2).

表 2 表层沉积物 $\mathrm{C} 、 \mathrm{~N} 、 \mathrm{H}$ 和 $\mathrm{O}$ 元素组成及 DOM 紫外-可见光谱参数

Tab.2 Composition of C, N, H and O elements and UV-vis spectral parameters of DOM in surface sediment

\begin{tabular}{|c|c|c|c|c|c|c|c|c|c|c|c|c|}
\hline \multirow{2}{*}{ 采样点 } & \multicolumn{4}{|c|}{ 元素含量/\% } & \multicolumn{4}{|c|}{ 摩尔比 } & \multicolumn{2}{|c|}{ 疏水性 DOM } & \multicolumn{2}{|c|}{ 亲水性 DOM } \\
\hline & $\mathrm{C}$ & $\mathrm{N}$ & $\mathrm{H}$ & $\mathrm{O}$ & $\mathrm{H} / \mathrm{C}$ & $\mathrm{O} / \mathrm{C}$ & $(\mathrm{N}+\mathrm{O}) / \mathrm{C}$ & $\mathrm{O} / \mathrm{H}$ & $\mathrm{E}_{2} / \mathrm{E}_{3}$ & $\mathrm{~A}_{240-400}$ & $\mathrm{E}_{2} / \mathrm{E}_{3}$ & $\mathrm{~A}_{240-400}$ \\
\hline $\mathrm{S} 1$ & 4.88 & 0.31 & 0.96 & 9.78 & 2.36 & 1.50 & 1.56 & 0.64 & 24.05 & 13.59 & 7.33 & 8.68 \\
\hline S2 & 5.66 & 0.33 & 1.07 & 11.11 & 2.27 & 1.47 & 1.52 & 0.65 & 22.10 & 13.18 & 11.91 & 12.54 \\
\hline S3 & 5.14 & 0.31 & 1.15 & 10.23 & 2.68 & 1.49 & 1.54 & 0.56 & 7.55 & 8.34 & 9.36 & 15.75 \\
\hline S4 & 5.33 & 0.30 & 1.12 & 14.98 & 2.52 & 2.11 & 2.16 & 0.84 & 7.48 & 5.69 & 19.28 & 13.84 \\
\hline S5 & 3.21 & 0.21 & 1.09 & 14.63 & 4.07 & 3.42 & 3.48 & 0.84 & 14.99 & 7.64 & 12.57 & 18.49 \\
\hline S6 & 4.95 & 0.28 & 1.11 & 15.86 & 2.69 & 2.40 & 2.45 & 0.89 & 15.42 & 7.58 & 35.35 & 32.35 \\
\hline S7 & 4.33 & 0.24 & 0.95 & 13.25 & 2.63 & 2.30 & 2.34 & 0.87 & 11.62 & 3.75 & 64.98 & 33.69 \\
\hline S8 & 1.87 & 0.16 & 0.88 & 8.33 & 5.65 & 3.34 & 3.41 & 0.59 & 44.90 & 8.53 & 97.32 & 27.90 \\
\hline
\end{tabular}

不同点位沉积物 DOM 疏水性组分和亲水性组分紫外-可见光谱特征见图 4 和表 2. 特征值 $\mathrm{E}_{2} / \mathrm{E}_{3}$ 比是有 机质在 250 和 $365 \mathrm{~nm}$ 处吸光度的比值, 该参数能够反映有机质的腐殖化、团聚化程度和分子量大小. 当 $\mathrm{E}_{2}$ / $\mathrm{E}_{3}$ 值 $<3.5$ 时, 有机质组成以胡敏酸为主, 而 $\mathrm{E}_{2} / \mathrm{E}_{3}$ 值 $>3.5$ 时, 有机质组成则以富里酸为主, 并且 $\mathrm{E}_{2} / \mathrm{E}_{3}$ 值与有 
机质的分子量呈反比 ${ }^{[33-34]}$. $\mathrm{A}_{240 \sim 400}$ 值是 DOM 样品在 240 400 nm 波段的积分面积, 主要与电子传递带有关, 电子传递的强度受极性官能团的影响较大, 而不受苯环存在的影响. 当有机质芳香环上存在羧基、羰基和羟 基等官能团时, 其电子传递的强度增加, 而苯环上有脂肪族结构时, 则不会提高其电子传递强度 ${ }^{[33]}$. 不同点 位沉积物疏水性 DOM 的 $\mathrm{E}_{2} / \mathrm{E}_{3}$ 和 $\mathrm{A}_{240-400}$ 分别变化在 7.48 44.90 和 3.75 13.59 之间, 其中 $\mathrm{E}_{2} / \mathrm{E}_{3}$ 则表现出 南北高、中部低的变化趋势,而 $\mathrm{A}_{240-400}$ 呈现出北部高、南部低的变化趋势 (表 2). 不同点位沉积物亲水性 DOM 的 $\mathrm{E}_{2} / \mathrm{E}_{3}$ 和 $\mathrm{A}_{240-400}$ 分别变化在 7.33 97.32 和 8.68 33.69 之间,二者均表现出南部高、北部低的变化趋 势. 表明由北至南亲水性 DOM 腐殖化水平和电子传递强度升高, 分子量降低, 但疏水性 DOM 的腐殖化水平 和电子传递强度下降, 分子量则表现出南北低、中间高的特点. 相比较而言, 北部点位 S1 和 S2 沉积物 DOM 的 $\mathrm{E}_{2} / \mathrm{E}_{3}$ 和 $\mathrm{A}_{240-400}$ 值均表现为: 疏水性 DOM> 亲水性 DOM, 而中部和南部点位 $\mathrm{S} 3 \sim \mathrm{S} 8$ 沉积物 DOM 的 $\mathrm{E}_{2} / \mathrm{E}_{3}$ 和 $A_{240-400}$ 值则表现为疏水性 DOM<亲水性 DOM.
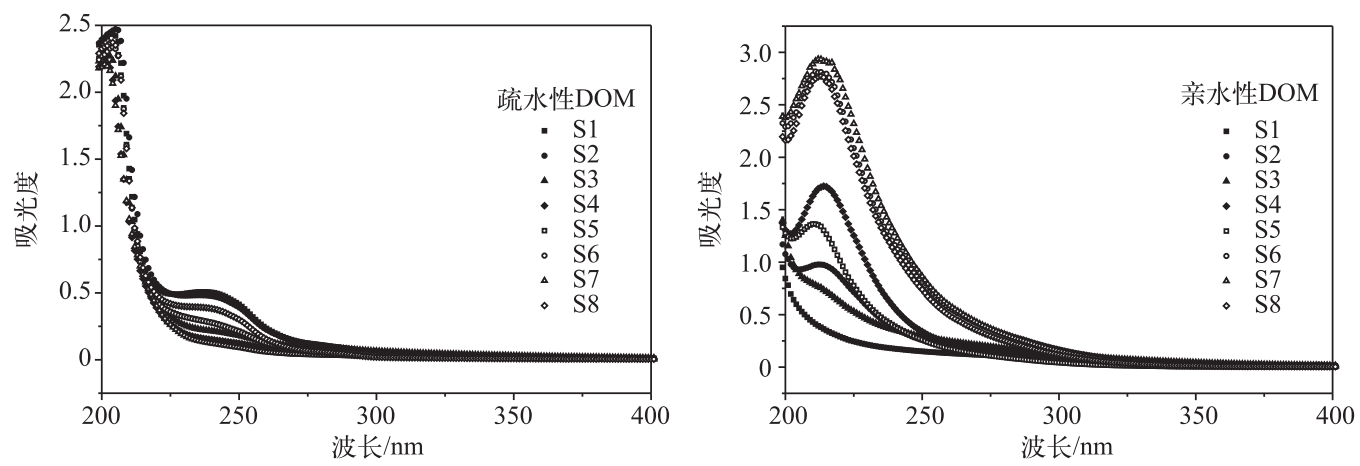

图 4 沉积物疏水性和亲水性 DOM 紫外-可见光谱特征

Fig.4 UV-vis spectrogram of hydrophobic and hydrophilic DOM in surface sediment

\section{3 沉积物磷形态与有机质组成结构之间的关系}

沉积物不同形态磷含量与 $C 、 N 、 H$ 和 $O$ 元素组成及 DOM 光谱特征参数密切相关 (表 3 ), 不同点位沉积 物 $\mathrm{NH}_{4} \mathrm{Cl}-\mathrm{P}$ 含量与 $\mathrm{C} 、 \mathrm{~N}$ 和 $\mathrm{H}$ 含量之间呈显著正相关 $(n=8, P<0.01$ 或 $P<0.05)$, 表明随着沉积物有机质含 量的增加, $\mathrm{NH}_{4} \mathrm{Cl}-\mathrm{P}$ 含量显著升高. $\mathrm{NH}_{4} \mathrm{Cl}-\mathrm{P}$ 是磷酸盐通过静电引力吸附在沉积物表面的磷形态, 主要包括 间隙水中磷、 $\mathrm{CaCO}_{3}$ 结合的磷, 以及沉积的植物碎屑中菌体细胞降解析出的磷, 这部分磷在沉积物一水界面是 不稳定的, 通常在扰动等外力条件下很容易释放到水体 ${ }^{[4,29]}$. 前人研究表明 ${ }^{[4,7-9]}, \mathrm{NH}_{4} \mathrm{Cl}-\mathrm{P}$ 含量与沉积物有 机质及钻含量密切相关, 随着沉积物 TOC 和 $\mathrm{CaCO}_{3}$ 含量的升高, $\mathrm{NH}_{4} \mathrm{Cl}-\mathrm{P}$ 含量显著增加, 这与本研究结果一 致. 本研究 $\mathrm{NH}_{4} \mathrm{Cl}-\mathrm{P}$ 含量与 $\mathrm{H} / \mathrm{C} 、 \mathrm{O} / \mathrm{C}$ 和 $(0+\mathrm{N}) / \mathrm{C}$ 摩尔比则呈显著负相关 $(n=8, P<0.01$ 和 $P<0.05)$. 由于 $\mathrm{H} / \mathrm{C}$ 摩尔比能够反映脂肪族和芳香族化合物的多少,而 $\mathrm{O} / \mathrm{C}$ 和 $(\mathrm{O}+\mathrm{N}) / \mathrm{C}$ 摩尔比与芳香环上差基、羒基和羰 基等官能团结构有关. 因此, 沉积物 $\mathrm{NH}_{4} \mathrm{Cl}-\mathrm{P}$ 不仅与有机质含量有关, 还受有机质组成结构影响. 此外, $\mathrm{NH}_{4} \mathrm{Cl}-\mathrm{P}$ 含量与亲水性 DOM 光谱参数 $\mathrm{E}_{2} / \mathrm{E}_{3}$ 之间呈显著负相关 $(n=8, P<0.05)$, 而与疏水性 DOM 相关性 不显著 $(n=8, P>0.05)$. 由于 $\mathrm{E}_{2} / \mathrm{E}_{3}$ 与 DOM 分子量呈反比, 因此, 沉积物 $\mathrm{NH}_{4} \mathrm{Cl}-\mathrm{P}$ 含量随着亲水性 DOM 分 子量的增大显著增加,而疏水性 DOM 对 $\mathrm{NH}_{4} \mathrm{Cl}-\mathrm{P}$ 含量的影响不大. 由于亲水性 DOM 通常含有更多的差基、 羧基和羰基等极性官能团结构, DOM 亲水性基团与磷酸盐竞争吸附或阴离子替换作用很可能是导致 $\mathrm{NH}_{4} \mathrm{Cl}-\mathrm{P}$ 含量降低的主要原因 ${ }^{[18-19]}$.

很多研究认为,沉积物 BD-P 和 NaOH-rP 含量主要与铁铝氧化物或氢氧化物有关 ${ }^{[10-12]}$. Kopacek 等 ${ }^{[10]}$ 研究发现, 当 $\mathrm{Al}_{\mathrm{NaOH} ~ 25}: \mathrm{Fe}_{\mathrm{BD}}$ 摩尔比 $>3, \mathrm{Al}_{\mathrm{NaOH} \sim 25}: \mathrm{P}_{(\mathrm{H}, 0+\mathrm{BD})}$ 摩尔比 $>25$ 时, 沉积物 BD-P 含量显著下降, 反之, 沉 积物 BD-P 含量显著增加. 尽管如此, 当 $\mathrm{Al}_{\mathrm{NaOH} \sim 25}: \mathrm{P}_{(\mathrm{H}, 0+\mathrm{BD})}$ 摩尔比 $<25$ 时, BD-P 随着溶解性有机碳含量 $\mathrm{OC}_{(\mathrm{BD}+\mathrm{H}, 0)}$ 的增加呈现线性增加趋势 $\left(R^{2}=0.96, P<0.001\right)$, 表明在氢氧化铝含量较低的沉积物中, BD-P 的增 加显著受沉积物有机质增加的影响. 本研究发现 $\mathrm{NaOH}-\mathrm{rP}$ 和 BD-P $+\mathrm{NaOH}-\mathrm{rP}$ 含量均与 $\mathrm{O}$ 含量及 $\mathrm{O} / \mathrm{H}$ 摩尔 比呈显著负相关, 表明随着沉积物有机质氧化度升高、极性增强和疏水性减弱, 沉积物铁铝氧化物对磷的吸 
附能力下降. 另外, $\mathrm{NaOH}-\mathrm{rP}$ 和 BD-P $+\mathrm{NaOH}-\mathrm{rP}$ 和 HCl-P 含量均与疏水性 DOM 的 $\mathrm{A}_{240-400}$ 呈显著正相关 $(n=$ $8, P<0.05)$, 但与亲水性 DOM 的 $\mathrm{A}_{240-400}$ 之间相关性不显著 $(n=8, P>0.05)$. 表明沉积物 BD-P、NaOH-rP 和 HCl-P 含量主要与疏水性 DOM 苯环上羟基、羧基和羰基等官能团结构有关, 而受亲水性 DOM 影响较小. 前 人研究发现, 低分子量有机酸能够活化结晶铁铝氧化物和阻碍无定形铁铝氧化物结晶, 从而促进了磷的吸 附作用 ${ }^{[16]}$, 土壤中磷吸附能力的提高与有机质分解产生低分子量有机化合物通过与金属键合, 形成具有稳 定化学结构的有机一金属螯合体有关 ${ }^{[17]}$.Sindelar 等研究 ${ }^{[36]}$ 发现, 随着天然有机质 (NOM) 含量的增加, 磷在 方解石表面的共沉淀最大表面积也显著增大. 因此, 疏水性 DOM 基团与铁、铝和钻矿物的键合作用增强了磷 酸盐在沉积物上的吸附和共沉淀作用, 进而提高了 BD-P、 NaOH-rP 和 HCl-P 含量. 另外, NaOH-nrP 与 $\mathrm{N}$ 含量 呈显著正相关, 但与 $\mathrm{O} / \mathrm{C}$ 和 $(\mathrm{O}+\mathrm{N}) / \mathrm{C}$ 摩尔比呈显著负相关 $(n=8, P<0.05)$, 由于磷酸单酯、磷酸二酯焦磷 酸和多聚磷酸等有机磷化合物是 $\mathrm{NaOH}-\mathrm{nr}$ 的主要来源 ${ }^{[28]}$, 表明 $\mathrm{NaOH}-\mathrm{nrP}$ 含量主要与有机质中有机磷组 成结构有关.

表 3 表层沉积物磷形态与 $\mathrm{C} 、 \mathrm{~N} 、 \mathrm{H}$ 和 O 元素组成及 DOM 紫外-可见光谱参数之间的关系

Tab.3 Relationships between $\mathrm{P}$ fractions and composition of $\mathrm{C}, \mathrm{N}, \mathrm{H}$ and

$\mathrm{O}$ elements and UV-vis spectral parameters of DOM in surface sediment

\begin{tabular}{|c|c|c|c|c|c|c|c|}
\hline & $\mathrm{NH}_{4} \mathrm{Cl}-\mathrm{P}$ & BD-P & $\mathrm{NaOH}-n r P$ & $\mathrm{NaOH}-\mathrm{rP}$ & $\mathrm{BD}-\mathrm{P}+\mathrm{NaOH}-\mathrm{rP}$ & HCl-P & Res-P \\
\hline C & $0.931^{* *}$ & 0.557 & 0.698 & -0.259 & -0.073 & 0.075 & 0.165 \\
\hline $\mathrm{N}$ & $0.954^{* *}$ & 0.644 & $0.731^{*}$ & -0.029 & 0.161 & 0.263 & 0.359 \\
\hline $\mathrm{H}$ & $0.737^{*}$ & -0.096 & 0.257 & -0.217 & -0.224 & -0.481 & 0.210 \\
\hline $\mathrm{O}$ & 0.137 & -0.117 & -0.191 & $-0.751^{*}$ & $-0.713^{*}$ & -0.660 & -0.607 \\
\hline $\mathrm{H} / \mathrm{C}$ & $-0.817^{*}$ & -0.605 & -0.587 & 0.284 & 0.081 & -0.111 & -0.074 \\
\hline $\mathrm{O} / \mathrm{C}$ & $-0.850^{* *}$ & -0.644 & $-0.752^{*}$ & -0.104 & -0.281 & -0.425 & -0.430 \\
\hline$(\mathrm{N}+\mathrm{O}) / \mathrm{C}$ & $-0.852^{* *}$ & -0.641 & $-0.752^{*}$ & -0.095 & -0.272 & -0.418 & -0.424 \\
\hline $\mathrm{O} / \mathrm{H}$ & -0.143 & -0.097 & -0.318 & $-0.804^{*}$ & $-0.756^{*}$ & -0.577 & -0.796 * \\
\hline $\mathrm{E}_{2} / \mathrm{E}_{3}{ }^{1)}$ & -0.607 & 0.007 & -0.168 & 0.468 & 0.425 & 0.489 & 0.031 \\
\hline $\mathrm{A}_{240-400}{ }^{1)}$ & 0.320 & 0.657 & 0.447 & $0.717^{*}$ & $0.839^{* *}$ & $0.814^{*}$ & 0.641 \\
\hline $\mathrm{E}_{2} / \mathrm{E}_{3}{ }^{2)}$ & $-0.770^{*}$ & -0.465 & -0.400 & -0.194 & -0.311 & -0.147 & -0.537 \\
\hline $\mathrm{A}_{240-400}{ }^{2)}$ & -0.571 & -0.565 & -0.520 & -0.510 & -0.626 & -0.542 & -0.796 * \\
\hline
\end{tabular}

1) 代表疏水性 DOM,2) 代表亲水性 DOM.

\section{3 结论}

1) 阳宗海表层沉积物不同形态磷含量总体呈现北部高、南部低的变化趋势. 其中沉积物 Mobile-P 含量 在 $68.67 \sim 124.70 \mathrm{mg} / \mathrm{kg}$ 之间变化, 平均占 TP 含量的 $9.81 \%$, 表现为 $\mathrm{BD}-\mathrm{P}>\mathrm{NaOH}-\mathrm{nrP}>\mathrm{NH}_{4} \mathrm{Cl}-\mathrm{P}$; 沉积物 Immobile-P 含量在 $496.73 \sim 908.28 \mathrm{mg} / \mathrm{kg}$ 之间变化,平均占 TP 含量的 $60.86 \%$.

2) 阳宗海表层沉积物 $C 、 N$ 含量和疏水性 DOM 光谱参数 $A_{240-400}$ 表现出北部高、南部低的变化趋势, 但 $\mathrm{H} / \mathrm{C} 、 \mathrm{O} / \mathrm{C}$ 和 $(\mathrm{N}+\mathrm{O}) / \mathrm{C}$ 和亲水性 $\mathrm{DOM}$ 光谱参数 $\mathrm{E}_{2} / \mathrm{E}_{3}$ 和 $\mathrm{A}_{240-400}$ 变化趋势与之恰好相反. 表明阳宗海由北至 南沉积物有机质含量下降, 疏水性 DOM 官能团减弱, 但沉积物脂肪族化合物及芳香环上具有羰基、羟基和 羧基或氨基和硝基等官能团结构化合物增多,亲水性 DOM 官能团增强.

3) 沉积物 $\mathrm{NH}_{4} \mathrm{Cl}-\mathrm{P}$ 含量与 $\mathrm{C} 、 \mathrm{~N}$ 和 $\mathrm{H}$ 含量之间呈显著正相关, 但与 $\mathrm{H} / \mathrm{C} 、 \mathrm{O} / \mathrm{C} 、(\mathrm{~N}+\mathrm{O}) / \mathrm{C}$ 和亲水性 $\mathrm{DOM}$ 的 $\mathrm{E}_{2} / \mathrm{E}_{3}$ 呈显著负相关; $\mathrm{NaOH}-\mathrm{rP}$ 和 $\mathrm{BD}-\mathrm{P}+\mathrm{NaOH}-\mathrm{rP}$ 含量均与 $\mathrm{O}$ 含量及 $\mathrm{O} / \mathrm{H}$ 摩尔比呈显著负相关; $\mathrm{NaOH}-\mathrm{rP} 、 \mathrm{BD}-\mathrm{P}+\mathrm{NaOH}-\mathrm{rP}$ 和 HCl-P 含量均与疏水性 DOM 的 $\mathrm{A}_{240-400}$ 呈显著正相关,但与亲水性 DOM 的 $\mathrm{A}_{240-400}$ 相关性不显著, 表明天然有机质组成结构是影响沉积物磷赋存形态的重要因素.

\section{4 参考文献}

[ 1 ] Wang Chao, Zou Limin, Wang Peifang et al. Fractionation of phosphorus in suspended matter and sediment. Environmental 
Science, 2008, 29(5): 1303-1307. [王超, 邹丽敏, 王沛芳等. 典型城市浅水湖泊沉积物磷形态的分布及与富营养 化的关系. 环境科学, 2008, 29(5): 1303-1307.]

[ 2 ] Li Anding, Zhang Yi, Zhou Beihai et al. In-situ control technology of phosphorus in sediment of eutrophic lake. Acta Hydrobiologica Sinica , 2014, 38(2) : 370-374. [李安定, 张义, 周北海等. 富营养化湖泊沉积物磷原位控制技术. 水生生物学报, 2014, 38(2): 370-374.]

[ 3 ] Huang Qinghui, Wang Donghong, Wang Chunxia et al. Relation between phosphorus forms in the sediments and lake eutrophication. China Environmental Science, 2003, 23(6) : 24-27. [黄清辉, 王东红, 王春霞等. 沉积物中磷形态与湖 泊富营养化的关系. 中国环境科学, 2003, 23(6): 24-27.]

[ 4 ] Wang SR, Jin XC, Zhao HC et al. Phosphorus fractions and its release in the sediments from the shallow lakes in the middle and lower reaches of Yangtze River area in China. Colloids and Surfaces A: Physicochemical and Engineering Aspects, $2006,273(1)$ : 109-116.

[ 5 ] Meis S, Spears BM, Maberly SC et al. Assessing the mode of action of Phoslock @ in the control of phosphorus release from the bed sediments in a shallow lake (Loch Flemington, UK). Water Research, 2013, 47(13) : 4460-4473.

[ 6 ] Reitzel K, Hansen J, Andersen FØ et al. Lake restoration by dosing aluminum relative to mobile phosphorus in the sediment. Environmental Science \& Technology, 2005, 39(11): 4134-4140.

[ 7 ] Wang SR, Jin XC, Pang Y et al. Phosphorus fractions and phosphate sorption characteristics in relation to the sediment compositions of shallow lakes in the middle and lower reaches of Yangtze River region, China. Journal of Colloid and Interface Science, 2005, 289(2) : 339-346.

[ 8 ] Herlihy M, McGrath D. Phosphorus fractions and adsorption characteristics in grassland soils of varied soil phosphorus status. Nutrient Cycling in Agroecosystems, 2007, 77(1): 15-27.

[ 9 ] Kaiserli A, Voutsa D, Samara C. Phosphorus fractionation in lake sediments-Lakes Volvi and Koronia, N. Greece. Chemosphere, 2002, 46(8): 1147-1155.

[10] Kopacek J, Borovec J, Hejzlar J et al. Aluminum control of phosphorus sorption by lake sediments. Environmental Science \& Technology, 2005, 39(22) : 8784-8789.

[11] Igwe CA, Zarei M, Stahr K. Fe and Al oxides distribution in some ultisols and inceptisols of southeastern Nigeria in relation to soil total phosphorus. Environmental Earth Sciences, 2010, 60(5) : 1103-1111.

[12] Virtasalo JJ, Kotilainen AT. Phosphorus forms and reactive iron in lateglacial, postglacial and brackish-water sediments of the Archipelago Sea, northern Baltic Sea. Marine Geology, 2008, 252(1) : 1-12.

[13] Kleeberg A, Herzog C, Hupfer M. Redox sensitivity of iron in phosphorus binding does not impede lake restoration. Water Research, 2013, 47(3): 1491-1502.

[14] Tessier A. Sorption of trace elements on natural particles in oxic environments. Environmental Particles, 1992, (1): 425-453.

[15] Zhang H, Davison W, Miller S et al. In situ high resolution measurements of fluxes of $\mathrm{Ni}, \mathrm{Cu}, \mathrm{Fe}$, and Mn and concentrations of Zn and Cd in porewaters by DGT. Geochimica et Cosmochimica Acta, 1995, 59(20): 4181-4192.

[16] Wang C, Wang Z, Lin L et al. Effect of low molecular weight organic acids on phosphorus adsorption by ferric-alum water treatment residuals. Journal of Hazardous Materials, 2012, 203: 145-150.

[17] Guppy CN, Menzies NW, Moody PW et al. Competitive sorption reactions between phosphorus and organic matter in soil: A review. Soil Research, 2005, 43(2): 189-202.

[18 ] Sibanda HM, Young SD. Competitive adsorption of humus acids and phosphate on goethite, gibbsite and two tropical soils. Journal of Soil Science, 1986, 37(2): 197-204.

[19] Yan J, Jiang T, Yao Y et al. Preliminary investigation of phosphorus adsorption onto two types of iron oxide-organic matter complexes. Journal of Environmental Sciences, 2016, 42(6) : 152-162.

[20] Wang SR, Jin XC, Bu Q et al. Effects of particle size, organic matter and ionic strength on the phosphate sorption in different trophic lake sediments. Journal of Hazardous Materials, 2006, 128(2) : 95-105.

[21] Wang SR, Yi WL, Yang SW et al. Effects of light fraction organic matter removal on phosphate adsorption by lake sediments. Applied Geochemistry, 2011, 26(3) : 286-292.

[22] Rubinos DA, Iglesias L, Díaz-Fierros F et al. Interacting effect of $\mathrm{pH}$, phosphate and time on the release of arsenic from polluted river sediments (Anllóns River, Spain). Aquatic Geochemistry, 2011, 17(3) : 281-306. 
[23] Li Shiyu, Liu Bin, Yang Changliang et al. Effect of $\mathrm{pH}$ and total phosphorus concentration of overlying water on arsenic mobilization in the sediments containing high arsenic and iron salts. J Lake Sci, 2015, 27 (6) : 1101-1106. DOI: 10. 18307/2015.0615. [李世玉, 刘彬, 杨常亮等. 上覆水 $\mathrm{pH}$ 值和总磷浓度对含铁盐的高砷沉积物中砷迁移转化的影 响. 湖泊科学, 2015, 27(6): 1101-1106.]

[24] Wang Zhenhua, He Bin, Pan Xuejun et al. The levels, trends and risk assessment of arsenic pollution in Lake Yangzonghai, Yunnan. Scientia Sinica Chimica, 2011, 41(3) : 556-564. [王振华, 何滨, 潘学军等. 云南阳宗海砷污染水平、 变化趋势及风险评估. 中国科学: 化学, 2011, 41(3): 556-564.]

[25] Ruban V, López-Sánchez JF, Pardo P et al. Selection and evaluation of sequential extraction procedures for the determination of phosphorus forms in lake sediment. Journal of Environmental Monitoring, 1999, 1(1) : 51-56.

[26] Psenner R, Boström B, Dinka M et al. Fractionation of phosphorus in suspended matter and sediment. Archiv fuir Hydrobiologie Beiheft Ergebnisse der Limnologie, 1988, 30: 98-103.

[27 ] Liu HZ, Jeong J, Gray H et al. Algal uptake of hydrophobic and hydrophilic dissolved organic nitrogen in effluent from biological nutrient removal municipal wastewater treatment systems. Environment Science and Technology, 2012, 46(2): 713-721.

[28] Bai X, Ding S, Fan C et al. Organic phosphorus species in surface sediments of a large, shallow, eutrophic lake, Lake Taihu, China. Environmental Pollution, 2009, 157( 8) : 2507-2513.

[29] He Jia, Chen Chunyu, Deng Weiming et al. Distribution and release characteristics of phosphorus in water-sediment interface of Lake Dianchi. J Lake Sci, 2015, 27( 5) : 799-810. DOI: 10.18307/2015.0506. [何佳, 陈春瑜, 邓伟明等. 滇 池水-沉积物界面磷形态分布及潜在释放特征. 湖泊科学, 2015, 27 ( 5) : 799-810.]

[30] Yu F, Zong Y, Lloyd JM et al. Bulk organic $\delta^{13} \mathrm{C}$ and $\mathrm{C} / \mathrm{N}$ as indicators for sediment sources in the Pearl River delta and estuary, southern China. Estuarine, Coastal and Shelf Science, 2010, 87(4) : 618-630.

[31] Liu Wenli, Wu Jinggui, Zhao Xinyu et al. Combined humus and elemental composition in orchards soils of different cultivation years. Journal of Northest Forestry University, 2014, 42(6): 68-72. [刘文利, 吴景贵, 赵新宇等. 不同园龄果 园土壤腐殖质组分数量及其元素组成特征. 东北林业大学学报, 2014, 42(6): 68-72.]

[32] Steelink C. Implications of elemental characteristics of humic substances. In: Aiken GR, Mc Knight DM, Warshaw RL eds. Humic substances in soil, sediment, and water. New York: Johu Wiley Sons Inc, 1985: 457-476.

[33] Minero C, Lauri V, Falletti G et al. Spectrophotometric characterisation of surface lakewater samples: Implications for the quantification of nitrate and the properties of dissolved organic matter. Annali Di Chimica, 2007, 97( 10) : 1107-1116.

[34] Wang FL, Bettany JR. Influence of freeze-thaw and flooding on the loss of soluble organic carbon and carbon dioxide from soil. Journal of Environmental Quality, 1993, 22(4) : 709-714.

[35] He X, Xi B, Wei Z et al. Spectroscopic characterization of water extractable organic matter during composting of municipal solid waste. Chemosphere, 2011, 82(4) : 541-548.

[36] Sindelar HR, Brown MT, Boyer TH. Effects of natural organic matter on calcium and phosphorus co-precipitation. Chemosphere, 2015, 138: 218-224. 